\title{
The torso response element binds GAGA and NTF-1/Elf-1, and regulates tailless by relief of repression
}

\author{
Gwo-Jen Liaw, ${ }^{1}$ Karen M. Rudolph, ${ }^{1}$ Jian-Dong Huang, ${ }^{2}$ Todd Dubnicoff, ${ }^{2}$ Albert J. Courey, ${ }^{2,3}$ \\ and Judith A. Lengyel ${ }^{1,3,4}$ \\ ${ }^{1}$ Department of Molecular, Cell and Developmental Biology, ${ }^{2}$ Department of Chemistry and Biochemistry, and ${ }^{3}$ Molecular \\ Biology Institute, University of California, Los Angeles, Los Angeles, California 90095 USA
}

\begin{abstract}
Modulation of transcription factor activity leading to changes in cell behavior (e.g., differentiation versus proliferation) is one of the critical outcomes of receptor tyrosine kinase (RTK) stimulation. In the early Drosophila embryo, activation of the torso (tor) RTK at the poles of the embryo activates a phosphorylation cascade that leads to the spatially specific transcription of the tailless (tII) gene. Our analysis of the tor response element (tor-RE) in the tll promoter indicates that the key activity modulated by the tor RTK pathway is a repressor present throughout the embryo. We have mapped the tor- $\mathrm{RE}$ to an 11-bp sequence; using this sequence as the basis for protein purification, we have determined that the proteins GAGA and NTF-1 (also known as Elf-1, product of the grainyhead gene) bind to the tor-RE. We demonstrate that NTF-1 can be phosphorylated by MAPK (mitogen-activated protein kinase), and that $t$ ll expression is expanded in embryos lacking maternal NTF-1 activity; these results make NTF-1 a likely target for modulation by the tor RTK pathway in vivo. The data presented here support a model in which activation of the tor RTK at the poles of the embryos leads to inactivation of the repressor and therefore, to transcriptional activation (by activators present throughout the embryo) of the tll gene at the poles of the embryo.
\end{abstract}

[Key Words: Torso tyrosine kinase receptor; transcription factor; GAGA; NTF-1; Elf-1; tailless; Trithorax-like; grainyhead]

Received June 20, 1995; revised version accepted October 24, 1995.

A multitude of extracellular signals can lead to a change in the program of cellular gene activity. An important mechanism by which extracellular signals (growth factors) are transduced is by receptor tyrosine kinases (RTKs). Ligand stimulation of an RTK leads to activation of a series of proteins: Transient generation of Ras-GTP leads to activation of a cascade of serine-threonine $\mathrm{ki}$ nases in the order Raf-1, mitogen-associated protein (MAP) kinase kinase (MAPKK), and MAP kinase (MAPK) (for review, see Hill and Treisman 1995). This "cassette" of sequentially acting proteins functions in mammalian cultured cells in the platelet-derived growth factor (PDGF), epidermal growth factor (EGF), and nerve growth factor (NGF) activated pathways, in Drosophila in the sevenless and torso receptor-activated pathways, and in nematodes in the Let- 23 (an EGF receptor homolog)-activated pathway (for review, see Perrimon 1993).

Modification of the transcriptional program resulting from RTK activation can be ascribed, at least in part, to the function of activated MAPK in phosphorylating var-

${ }^{4}$ Corresponding author. ious ets family transcription factors (for review, see Hill and Treisman 1995). Although our understanding of the cassette of proteins involved in the phosphorylation cascade is fairly robust, the subsequent steps that ultimately lead to altered cell behavior are less well defined. Neither all of the transcription factors modulated, nor all of the genes targeted by any one activated RTK are known.

The terminal system in the early Drosophila embryo provides an apparently simple system in which to examine transcriptional modulations initiated by RTK activation. The defined components of the torso (tor) pathway are provided by maternal transcription. During early embryogenesis, generation of functional ligand at the poles of the embryo activates the tor RTK at the poles of the embryo, leading to activation of the kinases in the pathway and hence, to transcriptional activation of the genes tailless $(t l l)$ and huckebein $(h k b)$ in overlapping polar caps (Pignoni et al. 1990; Brönner and Jäckle 1991; for review, see Perrimon 1993). At the anterior of the embryo, $t l l$ and $h k b$ expression are modulated rapidly by the maternal bicoid and dorsal activities (Pignoni et al. 1992; Brönner et al. 1994), making it difficult to dissect out the relative contribution of the activated tor RTK. At 
the posterior of the embryo, however, tll and $h k b$ are regulated only by the the activated tor RTK, and the biological effect of tor RTK activation can be explained entirely by the transcriptional activation of these two genes, both of which encode transcription factors (Strecker et al. 1986; Pignoni et al. 1990, 1992; Weigel et al. 1990; Brönner et al. 1994).

The terminal system is one of the genetically bestcharacterized RTK systems. The roles in this pathway of the Drosophila Ras, Raf-1, MAPKK, and MAPK (ERKA) have been established by demonstrating interactions between mutations in the genes encoding these proteins with both loss-of-function and gain-of-function tor $\mathrm{mu}$ tations (for review, see Perrimon 1993). These tor mutations are invaluable reagents for evaluating the in vivo function of putative tor response elements in genes regulated by the pathway (Liaw and Lengyel 1992; Liaw et al. 19931.

Because the diversity of effects of activated RTKs might be attributable in part to the modulation of distinct constellations of transcription factors, identification of additional RTK-targetable transcription factors is of prime importance for our understanding of ligand-dependent alteration of cell function. Like the other components of the tor RTK-activated pathway, the transcription factors regulating tll transcription are almost certainly provided maternally (for review, see Perrimon 1993) and therefore, difficult to identify directly by genetic techniques. As an alternative biochemical approach, we have taken a first step toward identifying such transcription factors by mapping tor response elements (tor-REs), to which these proteins are predicted to bind, in the promoter of the target gene tll. Here, we report data supporting a model in which an 11-bp tor-RE mediates transcriptional repression (rather than activation) of the tll gene. Where the tor kinase pathway is activated at both poles of the embryo, this repression is lifted and transcriptional activation is brought about by activators most likely present throughout the embryo. As it is present in embryos, which can be obtained in large quantity, the tor system is biochemically accessible. We have purified two proteins from Drosophila embryo extracts, GAGA and NTF-1, that bind to the tor-RE; we discuss the possible in vivo roles of these proteins in regulating tll expression.

\section{Results}

\section{A tor responsive minimal tll regulatory module}

We initiated mapping of the tor-RE within a "minimal regulatory region" that drives expression in two polar caps in response to tor activation. This region was identified on the basis of previous work in which we used promoter-reporter fusion constructs to show that the regulation of $t 11$ expression by the activated terminal system is mediated by synergistically interacting proximal and distal regulatory regions (Liaw and Lengyel 1992). Within the proximal regulatory region (shown in Fig. 1) we identified a 121-bp fragment (region $\mathrm{D}_{3.2}$, composed of regions $\mathrm{P}, \mathrm{Q}$, and $\mathrm{R}$ in Fig. 1) that mediates regulation of expression by the tor RTK pathway (Liaw and Lengyel 1992; Liaw et al. 1993). Although a construct (G5) containing region $\mathrm{PQR}$ drives spatially correct expression, the level of lacZ expression is very low and can only be detected by in situ hybridization to the lac $Z$ mRNA (Liaw et al. 1993). Addition of the $60 \mathrm{bp}$ of region $O$ gives a regulatory fragment (region $\mathrm{O}-\mathrm{R}$, inserted into construct G11) that drives expression in the characteristic early tll pattern [i.e., in two symmetrical caps at the poles of the embryo [Fig. 1)]; the higher level of this expression is sufficient for further dissection analysis (see below). Region $\mathrm{O}-\mathrm{R}$ does not drive expression of the later appearing anterior stripe (that responds to bicoid and dorsal as well as tor regulation); elements responsible for this stripe have been mapped to other portions of the $t 11$ regulatory region (Liaw and Lengyel 1992). Region O-R does have the critical feature of mediating response to the tor gene; it does not drive expression at the poles of the embryo (terminal expression) in tor loss-of-function embryos $\left(\right.$ tor $\left.^{X R 1}\right)$, and it drives ectopic uniform expression in tor gain-of-function embryos $\left(\right.$ tor $^{D 4021}$ ) (data not shown). On the basis of the above characteristics, we define the 181-bp O-R region as a minimal regulatory region responding to tor RTK activation. Analysis of deletion mutants of this minimal regulatory region reveals that it contains elements that mediate activation throughout the embryo, as well as elements mediating spatially specific repression.

\section{Activator elements}

In addition to region $\mathrm{O}$ (mentioned above), regions $\mathrm{P}$ and $\mathrm{R} I$ (see Fig. 3, below) also contribute to the overall level of $t l l$ expression. When these regions are deleted or substituted, the overall level of expression is diminished throughout the embryo (constructs G11 and G16 in Fig. 1; construct G26 in Fig. 3 ). We presume that there are ubiquitous activator proteins that bind to sites within these regions and activate $t 11$ expression. Further analysis of these regions and of the proteins binding to them will be presented elsewhere (G.-J. Liaw, A. Gu, A.J. Courey, and J.A. Lengyel, in prep.).

\section{Spatially specific repression}

The spatially restricted $\operatorname{lac} Z$ terminal expression driven by construct G11 is brought about not by a spatially specific activation element, but rather by relief of repression. This is deduced by comparing expression driven by construct G11 (region OPQR, expression restricted to the poles of the embryo) to that driven by construct G14 (region OPQ, uniform expression) (Fig. 1B,C). Other construct pairs possessing and lacking region $\mathrm{R}$ [i.e., cf. G21 and G17 (OPR vs. OP) and cf. G16 with G18 (OQR vs. $\mathrm{OQ}$ ) give similar results (Fig. 1). The deletion of the $\mathrm{R}$ region results in uniform expression that resembles the expression pattern of the tll gene in tor gain-of-function embryos (Steingrimsson et al. 1991). The most reasonable interpretation of these data is that an element in the 
A

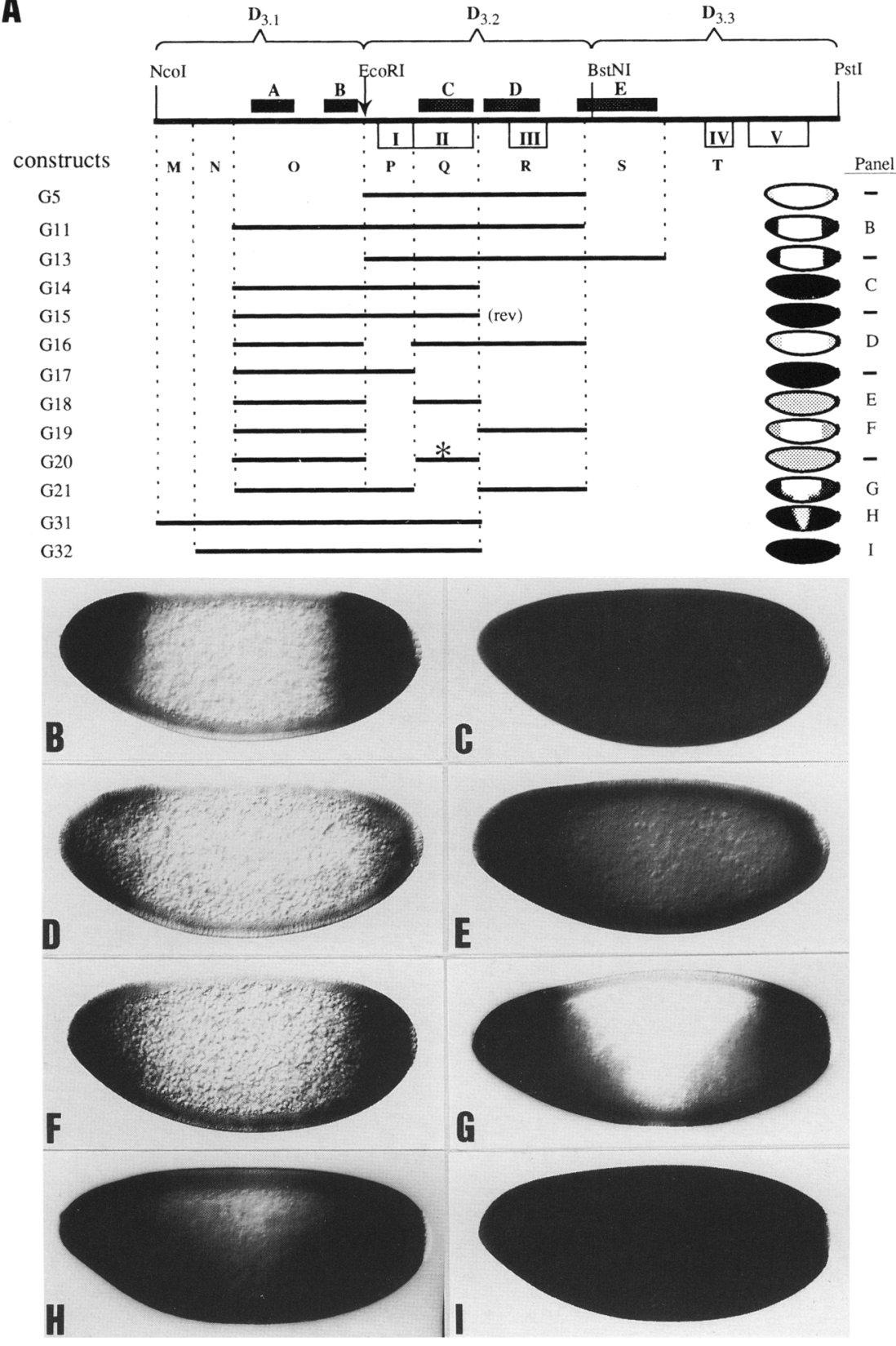

Figure 1. Dissection of the $t l l$ proximal promoter. (A) The 396-bp proximal regulatory region $\mathrm{D}_{3}$ has been described previously (Liaw et al. 1993). The solid boxes (AE) above the heavy line represent sites protected from DNase I digestion by embryonic nuclear extract; the open boxes below the line $(\mathrm{I}-\mathrm{V}\rangle$ represent DNA sequence conserved between $D$. melanogaster and $D$. virilis (Liaw et al. 1993). Patterns driven by the various $l a c Z$ reporter constructs (see Materials and methods) are shown in the cartoons at right. Construct G5 drives a correct, but low level, of terminal expression (Liaw et al. 1993). (B-I) The lac $Z$ mRNA expression patterns driven by constructs G11, G14, G16, G18, G19, G21, G31, and G32 are shown. Regions $D_{3.1}, D_{3.2}$, and $D_{3.3}$, described by Liaw et al. (1993), have been further subdivided into regions $M, N, O, P, Q, R, S$, and $T$ as indicated and are separated by vertical dotted lines. The asterisk in construct $\mathrm{G} 20$ indicates that the three tandem repeats in the $\mathrm{C}$ footprint (shown in Fig. 3C) have been mutagenized to the sequence ATATAGAAGCTTACTCTGC. All construct analyses shown in the cartoon embryos (right) are based on the comparisons of the lacZ RNA expression patterns in embryos at the early cellular blastoderm stage, determined by in situ hybridization. For constructs G14 and G18, $\beta$-galactosidase enzyme activity, measured as described previously (Liaw and Lengyel 19921, was fourfold higher for G14 as compared to Gl8. 57-bp $\mathrm{R}$ region mediates repression of transcription in the central domain of the embryo so that expression, driven by activators present throughout the embryo (see below 1 , is limited to the poles.

We localized two other weak repression elements, one in the $M$ region and one in the $Q$ region. The presence of a weak repression element in the $M$ region is revealed by a comparison of the expression driven by constructs G31 and $\mathrm{G} 32$; addition of the $M$ region leads to some repression in the center of the embryo (Fig. 1H,I). Because embryos carrying either G14 (possessing Q) or G17 (lacking Q) show uniform expression, it appears that the $Q$ region is not sufficient to repress central expression on its own. The presence of a weak repression element in the $Q$ re- gion is suggested, however, by the observations that (1) the expression domain driven by construct G21 (OPR) is slightly expanded relative to that driven by construct G11 (OPQR) (Fig. 1B,G), and (2) the expression level driven by construct $G 19(\mathrm{OR})$ is stronger than that driven by construct G16 (OQR) (Fig. 1D,F).

\section{The repressor elements can repress a heterologous promoter}

The above data strongly suggest that the region $M-R$ contains negative regulatory elements that repress $l a c Z$ expression in the central domain of the embryo. To further test this idea, we placed region $\mathbf{M}-\mathbf{R}$, oligomerized 
fourfold, upstream of a DNA fragment containing a portion of the Krüppel $(K r)$ promoter followed by a $h s p 70$ basal promoter element and the lac $Z$ gene (Hoch et al. 1990). The $K r-h s p 70$ promoter alone drives a strong central band of expression (Fig. 2A). When four copies of the $\mathrm{M}-\mathrm{R}$ region are inserted upstream of the $K r-h s p 70$ promoter, the central $K r$-promoter-driven stripe becomes both narrower and significantly weaker (Fig. 2B). In effecting repression from a position $2.5 \mathrm{~kb}$ from the $\mathrm{Kr}-$ hsp 70 promoter, the $M-\mathrm{R}$ region in this context displays one of the defining characteristics (repression from a distance) of a silencer.

Compared to the expression level driven by region M-R on its own (Fig. 2C), the terminal expression of

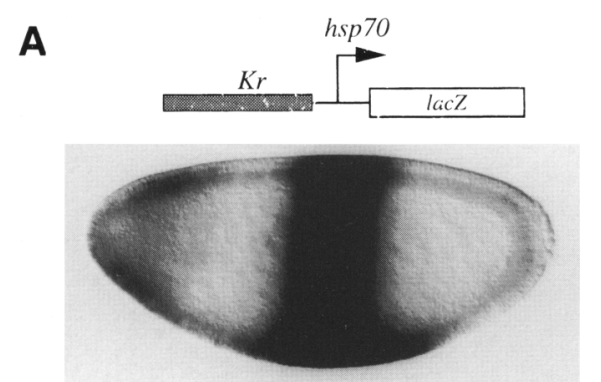

B

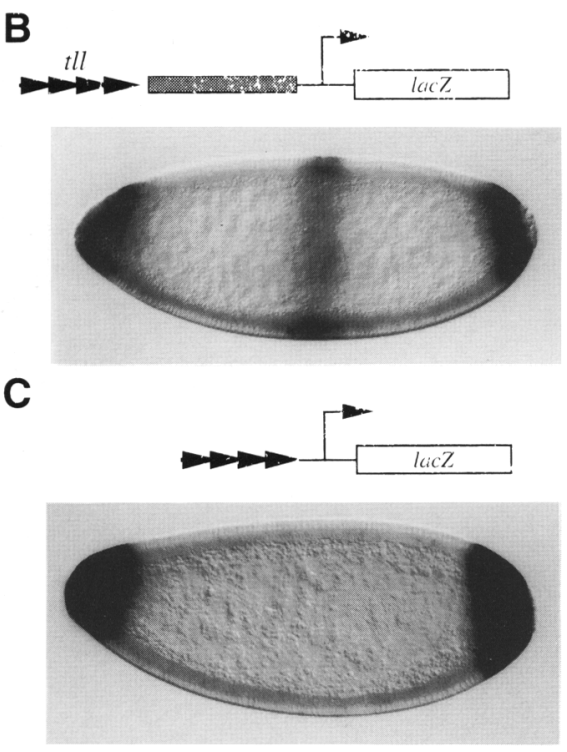

Figure 2. Negative regulatory elements in region $M-R$ repress a heterologous activator. $l a c Z$ mRNA distributions in embryos are revealed by in situ hybridization. $(A)$ Expression driven by the $K r-h s p 70 / l a c Z$ construct. The shaded box indicates $2.5 \mathrm{~kb}$ of the $K r$ promoter that drives strong expression in the middle of embryo (Hoch et al. 1990). (B) When the fourfold oligomerized $\mathrm{M}-\mathrm{R}$ region of the tll proximal promoter (Fig. $1 \mathrm{~A}$ ), indicated by four rightward-pointing arrows, is added to the $5^{\prime}$ end of the $K r$ promoter, expression in the middle of the embryo is reduced. The embryo shown in $B$ represents the intermediate level of repression seen with the indicated construct; of six transformant lines analyzed, two showed a stronger repression, three an equal repression, and one a weaker repression. (C) Expression driven by the oligomerized region $M-R$ alone. lac $Z$ is spatially correct but significantly weaker when the $K I$ promoter is added to region $M-\mathrm{R}$ (Fig. 2B). This weaker terminal expression may be attributable to the $t l l$ enhancers within region $M-R$ functioning less efficiently to activate $\operatorname{lac} Z$ transcription when they are moved further away from the transcriptional start site. Another possibility, not mutually exclusive, is that, as tll is known to repress $\mathrm{Kr}$ (Hoch et al. 1992; Steingrimsson 1992), Tailless protein present at the poles of the embryo might bind to elements in the $\mathrm{Kr}$ promoter and thus affect the ability of the adjacent $M-R$ repeats to drive terminal expression. If this latter interpretation is correct, then Tailless-binding elements must also function as silencer clements.

\section{Identification of the tor-RE}

Because removal of the 57-bp $\mathrm{R}$ region results in ectopic expression of $t 11$ throughout the embryo, a pattern similar to tll expression in tor gain-of-function embryos (Steingrimsson et al. 1991), the mechanism for regulation of $t$ ll expression by the tor RTK pathway is most likely to be relief of transcriptional repression. The repressor element mapped to the $\mathrm{R}$ region would then fulfill the definition of the tor-RE. Candidate sequences within the $\mathrm{R}$ region for the tor- $\mathrm{RE}$ are a 26-bp sequence protected from DNase I digestion by nuclear extract from early embryos (footprint $\mathrm{D}$ ) and an overlapping 28bp sequence (box III) conserved between Drosophila melanogaster and $D$. virilis, species that diverged at least 40 million years ago (see Fig. 1; Throckmorton 1975; Liaw et al. 19931.

To localize precisely the tor- $\mathrm{RE}$, the $\mathrm{R}$ region in construct Gll was mutagenized by linker scanning with a 14-bp sequence, dividing up the $\mathrm{R}$ region into four portions: R1, R2, R3, and R4 (see constructs G22-G26 in Fig. 3Al. Analysis of the expression pattern driven by these various mutagenized constructs in transformant embryos showed that the only mutation that had an effect on the spatially localized pattern of expression is that in construct G25 (Fig. 3C). The crucial result is that alteration of the $\mathrm{R} 2$ region results in strong derepression. The fact that the $\mathrm{R} 2$ region is located in the center of the $\mathrm{D}$ footprint, in the portion that overlaps with box III, supports the idea that the $\mathrm{R} 2$ region plays an important regulatory role.

Within the $D$. melanogaster and $D$. virilis tll regulatory regions, we identified five additional sequences that display close similarity to the sequence found in the R2 region. A consensus, TGCTCAATGAA, derived from these six sequences, is shown in Figure 4B. On the basis of the results of the mutagenesis described above, as well as additional experiments to be described below, we define the six sequences that share this 11-bp consensus as tor-REs. Two tor-REs are found in the proximal $D$. melanogaster tll regulatory region, one within footprint $\mathrm{D}$ (and analyzed by mutagenesis as described above), and another within region $M$, which (also as described above) contains a weak repression element (see Fig. 1). Two torREs are found in the distal tll regulatory region, in a 
A

constructs

G1
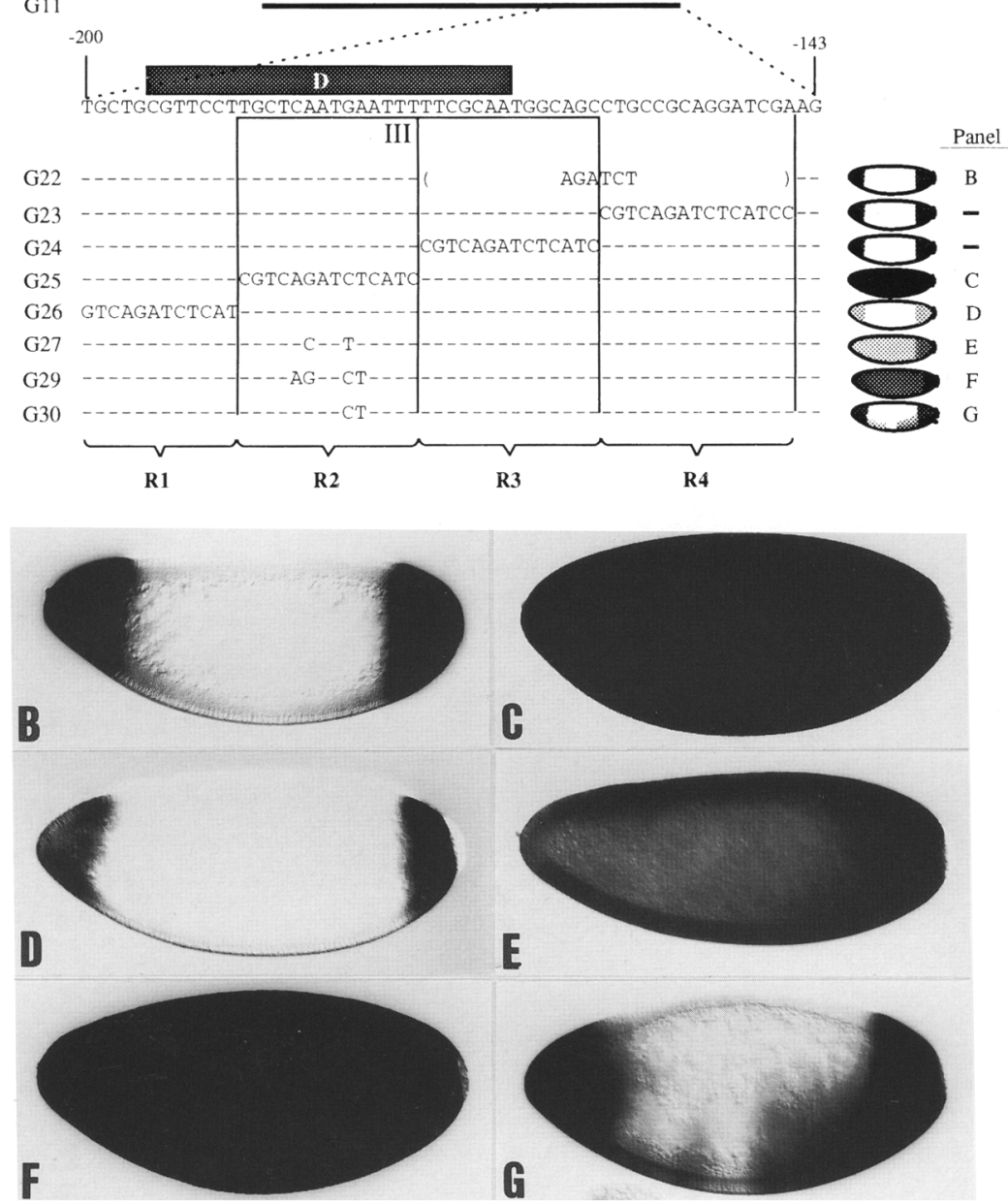

Figure 3. Mapping tor response elements in region $\mathrm{R}$ of the tll proximal promoter. The DNA sequence of region $R$ (from -143 to -200 l is shown. The shaded box indicates the position of footprint $\mathrm{D}$. The box III element is underlined. (A) Eight constructs (G22-G27, G29, and G30) derived from construct G11 by site-directed mutagenesis /see Materials and methods) are shown. The linker scanning mutagenesis (constructs G23-G26) divides region $\mathrm{R}$ into four subregions, R1, R2, R3, and R4. Patterns driven by the various constructs are shown in the cartoons at right. $(B-G) \operatorname{lac} Z$ mRNA expression driven by constructs G22, G25, G26, G27, G29, and G30, respectively. All construct analyses are based on the comparisons of the expression patterns of embryos at the early cellular blastoderm stage. 630-bp fragment (Fig. 4; Dm distal a and Dm distal b) that, like the PQR region, also drives terminal-specific expression (K.M. Rudolph, G.-J. Liaw, A.J. Courey, and J.A. Lengyel, in prep.). Like one of the tor-REs in the proximal promoter, one of the tor-REs in the distal promoter is also in the center of a sequence (footprint $\mathrm{F}$ ) protected from DNase I by embryonic nuclear extract (Fig. 4D). Two additional tor-REs are found in the proximal and distal $D$. virilis tll presumptive regulatory regions (Fig. 4; Dv proximal and Dv distall.

The tor-RE in the distal regulatory region was tested in experiments similar to those used to test the footprinted tor-RE in the proximal regulatory region. A 150-bp region containing box III sequence (which is protected by the $F$ footprint) was oligomerized and placed upstream of the lac $Z$ gene; this construct gives expression at the embryonic termini (Fig. 5B; construct K12). When the tor$\mathrm{RE}$ in the $\mathrm{K} 12$ construct was replaced with random nucleotide sequence (construct $\mathrm{K} 13$ ), repression was lost in the middle of embryo (Fig. 5C). [Although terminal expression of tll is not normally regulated by the dorso- ventral system (Pignoni et al. 1992), the exclusively ventral expression driven by constructs $\mathrm{K} 12$ and $\mathrm{K} 13$ is most likely attributable to activation by the Twist protein /see Fig. 5 legend).]

In summary, the experiments shown in Figures 3 and 5 show that the two tor-REs that can be footprinted, one in the proximal and one in the distal tll regulatory region, can be mutated independently to cause the terminal expression domains to expand, resulting in uniform expression along the anterior/posterior axis. These results support the notion that these sequence elements act as tor-REs.

To test the function of the proximal, footprinted tor$\mathrm{RE}$ in more detail, three mutant constructs with base substitutions in the 11-bp consensus sequence were generated (see Fig. 3A). Embryos carrying construct G29, with four base changes placed symmetrically in the center of the tor-RE, show an almost uniform expression (see Fig. 3F). Embryos transformed with the constructs G27 and G30, with only two base changes each, show expanded polar expression (i.e., less central repression); 
Figure 4. Consensus sequences in the distal and proximal tll promoters of both $D$. melanogaster and $D$. virilis. Box III sequences ( $\square$ below line) are found at several positions in the proximal and distal regulatory regions of both $D$. melanogaster $\langle A\rangle$ and $D$. virilis (this study, Liaw et al. 1993; G.-J. Liaw; data not shown). The positions of footprints of embryonic nuclear extract (from Liaw et al. 1993, and $D$, below) are indicated by solid boxes above the line $(A)$. Alignment of the box III-related sequences reveals an 11-bp consensus sequence (boldface type letters $\mid(B)$; on the basis of experiments described in the text, this consensus is referred to as the tor-RE consensus sequence. The significance of the 6-bp core sequence CTCAAT (arrows) found in some of the tor-REs is also discussed in the text. The tor-RE in region $M$ (which functions as a weak repressor element, see text) differs in 3 bp from the consensus tor-RE. Three repeats of the CTCAAT core sequence are also seen in footprint $\mathrm{C}(\mathrm{C})$. One of the tor-REs in the distal promoter region is also protected from DNase I digestion (indicated by box $\mathrm{F}$ in $A$ ) with embryonic nuclear extract $(D)$. A distal promoter DNA fragment $(-2926$ to -2298$)$ was $5^{\prime}$ labeled at the -2926 position and used as probe. The probe was sequenced using the Maxam and Gilbert sequencing reactions $\mathrm{C}+\mathrm{Y}$ (lane 1) and $\mathrm{G}+\mathrm{A}$ (lane 2), or incubated with no embryonic nuclear extract (lane 3), $5 \mu$ l of extract (lane 4) or $10 \mu \mathrm{l}$ of extract (lane 5). Similar experiments show that the tor-RE in the D. virilis proximal promoter is also protected from DNase I digestion by embryonic nuclear extract (data not shown).

the mutation in construct G30, however, has much less effect than that in construct G27 (Fig. 3E,G). These results implicate specific nucleotides within the 11-bp consensus sequence as being essential for the correct function of the tor-RE.

We identified a 6-bp core sequence, CTCAAT, within the tor-REs (see Fig. 4B). Only four of the six tor-REs [i.e., those containing a perfect match to the CTCAAT core sequence (indicated by arrows in Fig. 4B)], were protected from DNase I digestion by embryonic crude nuclear extract (Figs. 3A and 4D). In addition to the footprinted tor-REs, footprint $\mathrm{C}$ also contains three imperfect repeats of the CTCAAT core sequence. These results, summarized in Figure 4, suggest that the core sequence CTCAAT is crucial for protein binding to the tor-RE in vitro.

There are reasons to believe that the entire ll-bp torRE consensus sequence, not just the core sequence CT. CAAT contained within it, constitutes the tor-RE. When footprint $C$, which contains three repeats of the CTCAAT core sequence, is deleted from the minimal promoter, there is only a minor loss of transcriptional repression (see Fig. 1). In addition, making a change outside of the core sequence (GA to CT) does have an effect on repression (Fig. 3, construct G30).

In conclusion, our results show that the gene activa- tion mediated by the $t l$ promoter in response to activation of the tor RTK occurs by relief of repression. Within small regulatory regions that drive terminal expression of $t 11$, we have identified several elements that share an 11-bp consensus sequence, are footprinted by embryonic nuclear extract, and when mutated, cause relief of repression. These elements satisfy the definition of the tor-RE. The 6-bp core sequence, CTCAAT, may constitute a necessary, but not sufficient portion of the tor-RE.

\section{Purification and identification of two candidate tor-RE-binding proteins}

As described above (see Fig. 4) and in previous work (Liaw et al. 1993), there is an activity in crude embryonic nuclear extract that footprints the tor-RE; this activity appears equally concentrated in both 0 - to 4 - and 0 - to 12-hr extracts (G.-J. Liaw, data not shown). The first step in the purification of the tor-RE-binding protein /torREB) was the application of nuclear extract from 0 - to 12-hr embryos to heparin-Sepharose. Fractions eluting at $0.2 \mathrm{M}, 0.3 \mathrm{M}$, and $0.5 \mathrm{M} \mathrm{KCl}$ were assayed for specific tor-RE footprinting activity by using probes containing the wild-type tor-RE (construct G11, Fig. 1A) and the mutated tor-RE (construct G29, Fig. 3A). Based on the ability to footprint the wild type, but not the mutant 
A

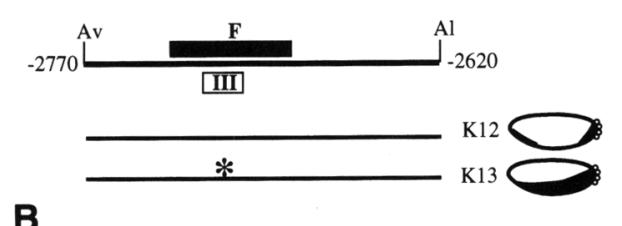

B

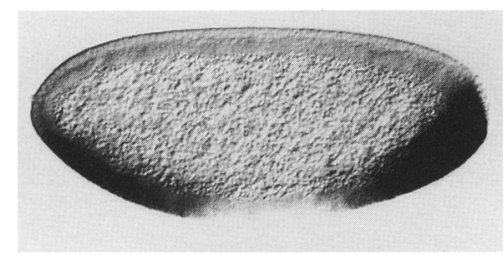

C

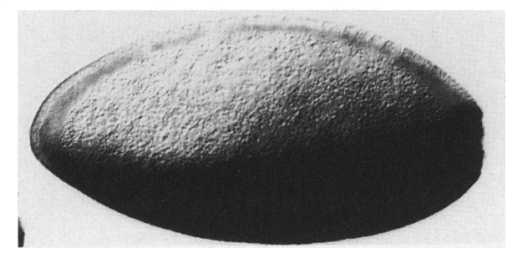

Figure 5. A tor-RE in the distal tll promoter. $(A \mid$ Schematic drawing of the $D$. melanogaster distal region (top line, see Fig. $3 \mathrm{~A}$ ). Footprint $\mathrm{F}$ is shown as a black box, with the tor-RE (box III) shown beneath (restriction sites: (Av) AvaI; (Al) AluI|. Construct K12 contains four copies of the wild-type distal region (see Fig. 3). Construct K13 contains four copies of the distal region with the 16-bp centered around the tor-RE replaced by the random sequence GACTGCAGACTGTCAG / which contains an internal PstI site) using site-directed mutagenesis. For construct $\mathrm{K} 13$, the asterisk indicates the mutagenized tor-RE (box III) element. To the right of the constructs are cartoons of the lac $Z$ mRNA expression pattern, as seen at the early cellular blastoderm stage, summarized from the expression seen in whole embryos. $(B, C)$ Embryos at the cellular blastoderm stage carrying the constructs $\mathrm{K} 12$ and $\mathrm{K} 13$, respectively. The ventral expression of the $\mathrm{K} 12$ and $\mathrm{K} 13$ constructs is most likely attributable to activation by the Twist protein for the following reasons: the constructs drive no expression in embryos lacking the Dorsal protein (K.M. Rudolph, data not shown), which is required to activate expression of the twist gene on the ventral side of the embryo; within the -2770 to -2620 fragment of the distal region there are no Dorsal-binding sites but there are four E-box containing sites that can be footprinted by Twist protein (G.-J. Liaw, data not shown).

tor-RE, two distinct activities were identified that separated in the initial heparin-Sepharose chromatography. Subsequently, each activity was purified separately; we refer to these two proteins in what follows as tor $-\mathrm{REB}_{\mathrm{a}}$ and tor- $\mathrm{REB}_{\mathrm{b}}$.

tor-REB $B_{a}$ is the GAGA protein

One tor-RE footprinting activity was identified in the material eluting from heparin-Sepharose at $0.5 \mathrm{M} \mathrm{KCl}$. As indicated in the flowchart in Figure 6A, this material was further purified by CM Sepharose column chromatography, and finally DNA affinity chromatography using the tor-RE sequence (see Materials and methods).
Analysis of the protein bound by the final DNA-affinity column by SDS-gel electrophoresis revealed the major component to be a polypeptide with an apparent molecular mass of $65 \mathrm{kD}$ (Fig. 6B). This protein is recognized by an anti-GAGA antibody in a Western blot (Fig. 6B). Furthermore, both the tor- $\mathrm{REB}_{\mathrm{a}}$ and the purified bacterially expressed GAGA protein (see Materials and methods) give identical footprints on the tor-RE (Fig. 7A). We conclude that the tor- $\mathrm{REB}_{\mathrm{a}}$ is the GAGA protein.

tor-REB is the DRE-binding protein, which is NTF-1 (Elf-1)

A second tor-REB protein eluted from heparin-Sepharose at $0.3 \mathrm{M} \mathrm{KCl}$. This eluate also contains activity binding to the $d p p$ repression element (DRE), the subject of another investigation (Huang et al. 1995). tor-REB was found to copurify with the DRE-binding protein. Using the protocol developed for purification of the DRE-binding protein [except the final DNA affinity chromatography, for which the tor-RE was used (see Materials and methods)] we purified a second tor-REB protein. Because the DRE-binding protein has been shown to be identical to the Drosophila transcription factor NTF-1 (also known as Elf-1) (Huang et al. 1995), we compared footprinting by NTF-l and the purified protein we called tor-REB ${ }_{\mathrm{b}}$. Bacterially expressed GST-NTF-1 and tor$\mathrm{REB}_{\mathrm{b}}$ give identical footprints on the tor-RE (Fig. $7 \mathrm{~B}$ ). The tor- $\mathrm{REB}_{\mathrm{b}}$ (NTF-1 purified from embryos) shows a reduced ability to footprint the mutated tor-RE (i.e., it is unable to bind the region where the DNA sequence is altered, although it still binds to the flanking regions) (Fig. 7B). We conclude that the tor $-\mathrm{REB}_{\mathrm{b}}$ is NTF-1.

\section{NTF-1 is a substrate for MAPK (ERKA)}

Because the tor-RE is the cis-regulatory element responding to activation of the tor RTK pathway, the torREBs are candidate substrates of the MAPK activated by the terminal system, ERKA. Bacterially expressed ERKA is not active in vitro (W. Biggs and L. Zipursky, pers. comm.); therefore, we used the homologous mammalian protein, bacterially expressed ERK2 (Haystead et al. 1992). Bacterially expressed GAGA and NTF-1 were tested as potential substrates by incubation with $\left[\gamma^{-32} \mathrm{P} \mid \mathrm{ATP}\right.$ and activated ERK2. Figure 8 shows that NTF-1 (but not GAGA) is phosphorylated by ERK2. The molar ratio of ${ }^{32} \mathrm{P}$ incorporated into NTF-1, relative to that incorporated into myelin basic protein (which is phosphorylated on one threonine; Erickson et al. 1990), indicates that there is also one phosphate incorporated per NTF-1 molecule.

\section{Decreased NTF-1/Elf-1 activity results in expansion of tll expression}

The availability of mutants in Drosophila can often provide the opportunity to test the in vivo function of a protein identified biochemically. This is, in principle, possible for NTF- 1 and the GAGA protein, as mutations 
A

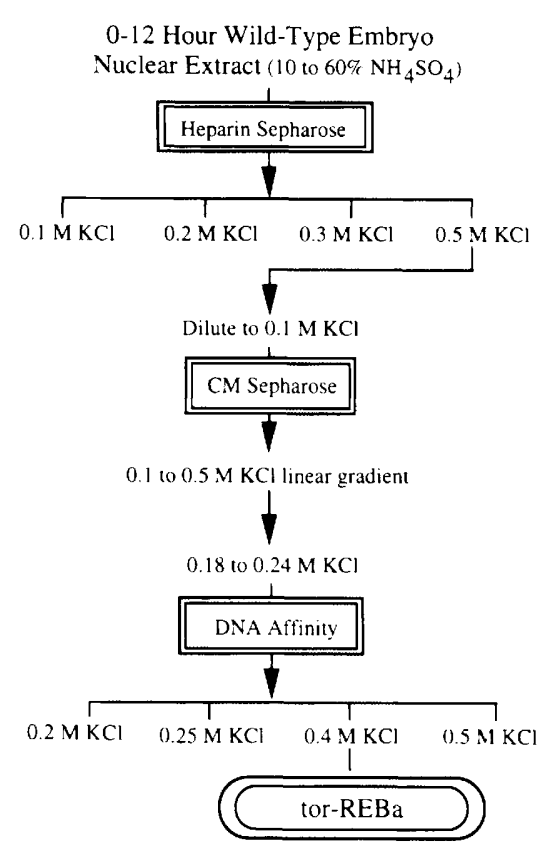

B

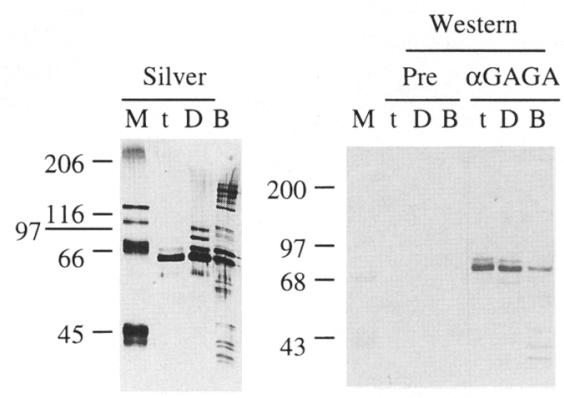

Figure 6. Purification and identification of the tor-REB $\mathrm{a}^{\text {. The purification }}$ scheme is shown in $A$ and described in more detail in Materials and methods. Purified tor- $\mathrm{REB}_{\mathrm{a}}(\mathrm{t})$ was compared to bacterially expressed and purified Drosophila GAGA $\{\mathrm{B}, \mathrm{D}$, respectively) by SDS-gel electrophoresis and immunoblotting. A silver-stained gel containing the various proteins is shown in $B$ (left). The apparent molecular mass of the purified tor- $\mathrm{REB}_{\mathrm{b}}$ is $65 \mathrm{kD}$; GAGA has been described previously as having an apparent molecular mass of $67 \mathrm{kD}$ (Soeller et al. 1993). On the right of $B$ are shown immunoblots of duplicate gels, probed with either preimmune serum (Pre) or with anti-GAGA antibody $(\alpha-G A G A)$. $(M)$ protein size markers in $\mathrm{kD}$. in the genes encoding these proteins have been identified. NTF-1 is encoded by the gene grainyhead $(g r h)$; loss-of-function grh mutations result in embryonic lethality with no obvious terminal defects (Bray and Kafatos 1991). The GAGA protein is encoded by the Trithorax-like (Trl) gene; loss-of-function $T r l$ mutations are not embryonic lethal, but do result in lethality during the third larval instar (Farkas et al. 1994).

Because the known components of the terminal system upstream of $t l l$ and $h k b$ are provided maternally (Perrimon 1993), and as tll transcription is activated very early in embryogenesis (i.e., by nuclear cycle 9; Pignoni et al. 1992), it is likely that any NTF-1 or GAGA protein involved in regulating initial expression of $t l l$ is provided for by maternal transcription. Analysis of ovaries and early embryos indicates that mRNAs for both proteins are indeed part of the maternal dowry (Soeller et al. 1993; Huang et al. 1995). Because null alleles of $g r h$ and $T r l$ are zygotic lethal, the effect of these mutations on early $t l l$ expression can best be tested by generating germ-line clones in heterozygous females. If either of these proteins plays a required role in repression of $t 11$, one would predict an expansion of tll expression in embryos from germ-line clones lacking expression of the particular protein.

Germ-line clones can be generated by X-irradiation of females carrying a dominant female sterile mutation, or by use of the recently developed $\mathrm{ovO}^{D}$-FLP-FRT system (Perrimon and Gans 1983; Chou et al. 1993). Using three different $T r l$ alleles and both the FLP-FRT system and $\mathrm{X}$-irradiation, however, we were unable to obtain an increase above background in the number of eggs laid by heterozygous $T r$ l/ovo ${ }^{D}$ females (G.-I. Liaw; data not shown). Therefore, it is likely that $\operatorname{Tr} l$ may be required for early events of oogenesis. For the weak allele $T_{r} 1^{13 C}$, homozygous escaper females can be obtained; embryos from these females show an approximately twofold decrease in level of tll expression, but no expansion in the tll expression domains (G.-J. Liaw; data not shown). Thus, at present, genetic experiments do not provide evidence supporting a requirement for GAGA in the regulation of tll expression.

Our ability to generate germ-line clones in heterozygous $g r h /+$ females allows a more definitive conclusion to be reached about the role of NTF- 1 in tll regulation. Although it was not possible to use the FRT-FLP system (because the insertion site of $\mathrm{P}\left[o v O^{D}\right]$ on the available FRT $o v O^{D}$ chromosome is a lethal allele of $g r h$ ), we were able to generate homozygous $g r h$ clones by X-irradiation of females of the genotype $g r h^{B 37} / F s(2) D$. A portion of the embryos from these $\mathrm{grh}^{B 37}$ germl-ine clones showed the predicted expansion of $t / l$ expression: There was a range in the size of the posterior cap of tll expression, from a domain covering $0 \%-20 \%$ egg length (no expansion, some of these embryos may have come from escaper $g r h / C y O$ females) to a domain covering $0 \%$ to $\sim 40 \%$ egg length (Fig. 9A,B). In no case was tll expression expanded to cover the entire embryo, as is seen in tor-dominant gain-of-function alleles (Steingrímsson et al. 1991). Interestingly, this expansion often appeared as a set of stripes rather than being continous (Fig. 9C); thus, there may be repressors of $t l l$ that are not distributed uniformly throughout the embryo.

In the above experiments, we were not able to distinguish between embryos lacking grh only maternally, or embryos lacking grh both maternally and zygotically. That the embryos showing expansion of tll expression indeed come from grh germ-line clones is indicated by two pieces of evidence: (1) expansion is not seen in embryos from unirradiated $g r h / F s(2) D$ females crossed to 
A

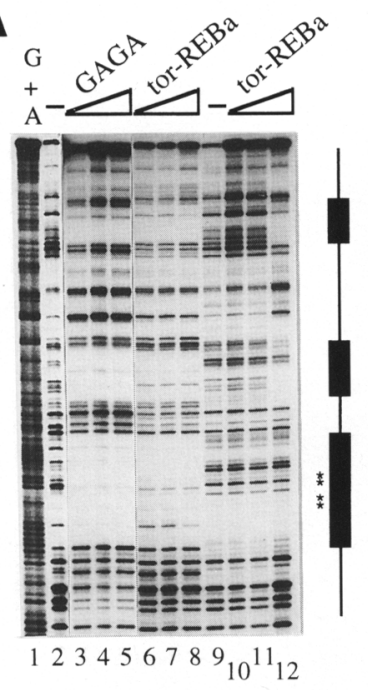

B

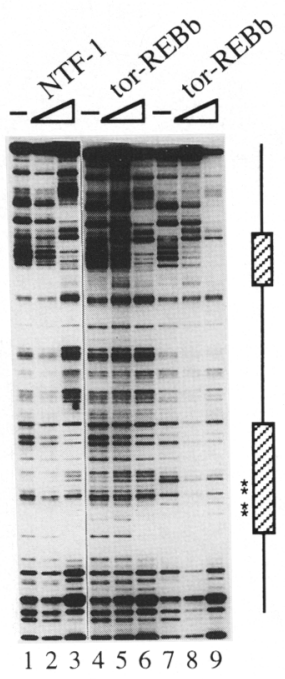

C

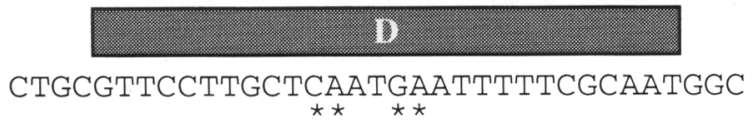

GAGA

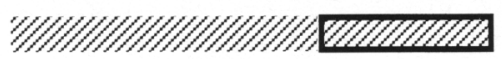

NTF-1

Figure 7. Comparison of the DNA-binding specificity between tor-REB ${ }_{a}$ and GAGA, and between tor-REB $b_{b}$ and NTF-1. The footprinting probe was either the wild-type tor-RE or a mutated version of the tor-RE that eliminates repression (see Fig. $3 \mathrm{~A}$ ). The binding to each of these probes by purified bacterially expressed GAGA (GAGA) is compared to that of purified tor- $\mathrm{REB}_{\mathrm{a}}$ in $A$, whereas the binding of purified bacterially expressed GSTNTF-1 (NTF-1) is compared to that of purified tor-REB $B_{\mathrm{b}}$ in $B$. Solid boxes in $A$ and hatched boxes in $B$ indicate the sites of footprints; asterisks indicate the location of substituted bases in the mutated tor-RE (the upper footprints in both $A$ and $B$ are to non-tor-RE sites). An alignment over the tor-RE of the GAGA footprint, the NTF-1 footprint, and the previously described footprint D (Liaw et al. 1993) is shown in C. At a low concentration, GAGA footprints only the left half of the D footprint (solid line); at higher concentrations, the GAGA footprint increases (shaded line). Similarly, NTF-1 at a low concentration footprints the right side of the D footprint (boxed hatching); at higher concentrations, the NTF-1 footprint increases /unboxed hatching $\mid$. Lane 1 in $A$ is a sequencing ladder; lanes 2-8 in $A$ and 1-6 in $B$ contain wild-type probe, and lanes $10-12$ in $A$ and 7-9 in $B$ contain mutant tor-RE. In $A, 1 \mu$ l (lanes 3, 6, 10), $2 \mu$ I (lanes $4,7,11$ ), and $5 \mu \mathrm{l}$ (lanes $5,8,12$ ) of solutions of GAGA and tor- $\mathrm{REB}_{\mathrm{a}}$ at 1 and $0.03 \mathrm{mg} / \mathrm{ml}$, respectively, were used. In $B, 1 \mu \mathrm{l}$ (lane 2) and $5 \mu \mathrm{l}$ (lane 3) of GST-NTF-1 at $0.1 \mathrm{mg} / \mathrm{ml}$, and 2 (lanes 5,8$)$ and $10 \mu \mathrm{l}$ (lanes 6,9) of the tor $-\mathrm{REB}_{\mathrm{b}}$ at $0.005 \mathrm{mg} / \mathrm{ml}$ (estimated), were used. "-" (lanes 2,9 in $A$, and lanes 1, 4, 7 in $B)$ indicates no protein added to the reaction mixture.

grh/CyO males, and (2) embryos from an inter se cross of $g r h^{B 37} /+$ do not show significant expansion of tll ex-

pression. Although we were unable to obtain germ-line clones using the $g r h^{B 32}$ allele, embryos from an inter se $g r h^{B .32} /+$ cross showed weak expansion of $t l l$ expression, always seen as two additional stripes rather than as a continuous domain (Fig. 9D). Thus, grh ${ }^{B 32}$ may be a stronger allele than $g r h^{B 37}$, indicated by its apparent maternal effect as a heterozygote and the fact that germ-line clones of the former allele were not obtained.

In conclusion, these genetic experiments are consistent with a required role for NTF-1 in the repression of tll.

\section{Discussion}

\section{tll regulation occurs by relief of repression}

Our in vivo promoter dissection has revealed that transcriptional activation of $t l l$ by the activated tor RTK pathway occurs by relief of repression. An 11-bp element, the tor-RE, is critical for this repression as mutagenesis of this element in the context of either the proximal or distal $t l l$ regulatory module results in expanded or, in some cases, uniform reporter gene expression. The finding that $t l l$ regulation involves relief from repression suggests the following model for how localized activation of the terminal system results in spatially specific $t l l$ transcription. In the central region of the embryo where the terminal system is not activated, repres-
A

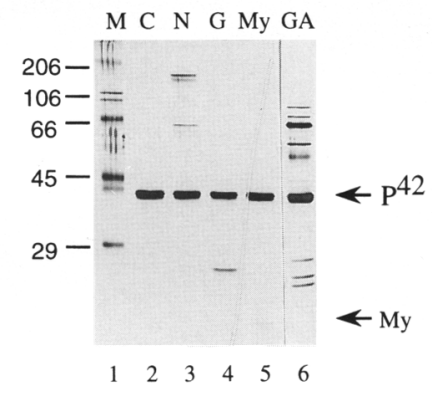

B

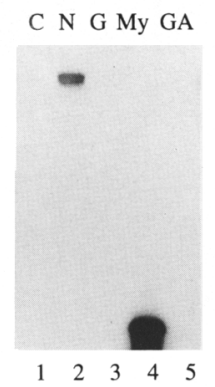

Figure 8. Phosphorylation of NTF-l by MAPK. Activated MAPK was prepared as described in Materials and methods, and incubated with GST-NTF-1 (N), GAGA (GA), either one of the control proteins GST $(\mathrm{G})$ or myelin basic protein $(\mathrm{My})$, or with no protein $(C)$. The reaction products were electrophoresed on an SDS-acrylamide gel and detected by silver staining $(A)$; an autoradiograph of this gel is shown in $B . M$ (lane 1 ) contains protein size markers. The activated MAPK $\left(\mathrm{P}^{42}\right)$ and $\mathrm{My}$ are indicated with arrows. Except for GAGA $(1 \mu \mathrm{g}), 0.1 \mu \mathrm{g}$ of each protein was added to the phosphorylation reaction. As determined by scanning of the gel, the ratio of dpm in the My doublet band to the dpm in the GST-NTF-1 band was 11:1. The molar ratio of My to GST-NTF-1 was 9:1 (because equal masses of My and GST-NTF-1 were loaded on the gel, and the relative molecular masses of these two proteins are 18.1 and $167 \mathrm{kD}$, respectively|. Because MAPK phosphorylates one site per My molecule (Erikson et al. 1990), these numbers suggest that under the reaction conditions used, MAPK also phosphorylates roughly one site per GST-NTF-1. 


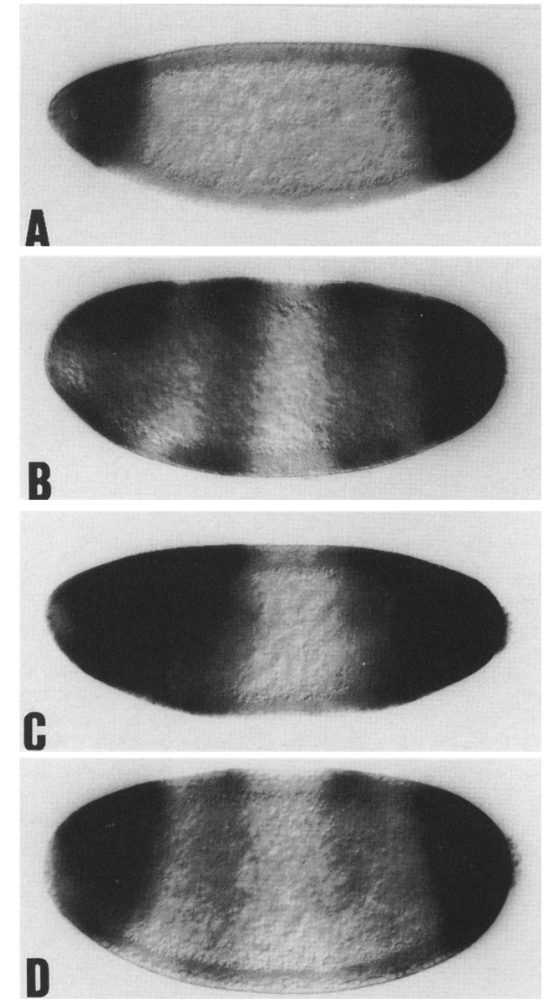

Figure 9. Loss of grh activity results in expansion of $t / l$ expression. Endogenous $t l l$ expression was detected by in situ hybrid. ization. The normal expression pattern is seen in a wild-type embryos $(A \mid$. Embryos from females carrying germ-line clones homozygous for the allele $g r h^{B 37}$ show expanded expression of $t l l(B, C)$. Embryos from a cross between $\mathrm{grh}^{B 32} /+$ heterozygous parents show a slightly expanded expression of $t l l(D)$.

sor proteins that interact with the tor-RE and perhaps with other DNA regulatory elements serve to silence the tll promoter. At the poles of the embryo, one or more of the proteins required for $t l l$ repression is modified and therefore, inactivated by the terminal system; this allows terminal-specific tll transcriptional activation by globally distributed activating factors. A prediction of this model is that one or more factors interacting with critical tll cis-regulatory elements (e.g., the tor-RE) will be found to be substrates of the terminal system phosphorylation cascade.

Various lines of evidence suggest that the tor-RE may silence transcription in an active manner rather than by the exclusion of activator proteins from overlapping binding sites. First, a region of the $t l l$ promoter containing the tor-RE is able to reduce the activity of the $K r$ enhancer at a distance. Second, deletion of the tor-RE from either the proximal or the distal regulatory module results in an expansion of the spatial domain in which transcription occurs, but does not result in a reduction in the level of transcription. If the tor-RE contained overlapping repressor- and activator-binding sites, then we would expect deletion of the tor-RE to result in a reduction in the overall level of expression.

\section{Multiple regulatory factors may contribute to tll repression}

Chromatographic fractionation of Drosophila embryo extracts allowed purification of two proteins that bind specifically to the tor-RE: GAGA and NTF-1. Because GAGA binds to the sequence TGAG (Kerrigan et al. 1991), it is not surprising that it also binds to the tor-RE, which contains TGAG on the opposite strand of the core sequence CTCAAT. The fact that mutations that render the tor- $\mathrm{RE}$ inactive also reduce or eliminate binding of GAGA suggests that this protein could contribute to $t 11$ repression. Although we have not been able to generate embryos completely lacking GAGA to test this idea in vivo, we note that, as GAGA is present in the freshly laid egg (Soeller et al. 1993), there is at least a potential for GAGA to play a role in tll expression.

Although there is no obvious motif shared between the previously reported NTF-1-binding site (Bray et al. 1989; Dynlacht et al. 1989) and the tor-RE that would explain the binding of NTF-1 to both of these sequences, a number of considerations suggest that NTF-1 plays a role in regulation of $t l l$ expression. First, as found for GAGA, mutations in the tor-RE that eliminate its repression function also reduce or eliminate NTF-1 binding. Second, NTF-1 mRNA is synthesized during oogenesis and appears to be transferred to the developing oocyte (Huang et al. 1995); therefore, it is likely that NTF-1 is present in the early embryo when transcription is activated during the syncytial blastoderm stage. Third, NTF-1 is phosphorylated in vitro by the mammalian MAPK homolog of ERKA, an essential protein kinase component of the terminal signaling cascade (Brunner et al. 1994b). Fourth, elimination of NTF-l activity from early embryos by the generation of germ-line clones results in an expansion of $t$ tl expression. We conclude that NTF-1 plays an essential role in the repression of $t l l$; it seems likely that its phosphorylation, as a result of tor RTK activation, diminishes its repressor activity.

Although it appears that NTF-1 (and possibly also GAGA) contributes to the repression of $t 11$, previous studies on NTF-1 and GAGA have focused on their roles as transcriptional activators. In vitro, NTF-1 binds to specific cis-regulatory elements in the Dopa decarboxylase, Ultrabithorax, and fushi tarazu promoters (Bray et al. 1989; Dynlacht et al. 1989) and acts as a transcriptional activator (Dynlacht et al. 1989; Attardi and Tjian 1993). GAGA binds in vitro to many regulatory regions, including those of various pattern formation, heat shock, and histone genes (for review, see Soeller et al. 1993); both biochemical and genetic studies suggest that GAGA, by modulating chromatin structure, allows access to the promoter of the basal transcriptional machinery and other activating transcription factors (Kerrigan et al. 1991; Lu et al. 1993; Tsukiyama et al. 1994; Farkas et al. 1994; O'Brien et al. 1995). Because a number of transcription factors have now been shown to be capable, in a context-dependent manner, of acting either as activators or repressors (for review, see by Roberts and Green 1995), it is reasonable to imagine that this might also be possible for NTF-1 and GAGA. 
Several pieces of evidence suggest that there are proteins additional to GAGA and NTF-1 that might be involved in regulation of $t$ ll. First, the expansion of $t 11 \mathrm{ex}-$ pression in NTF-1-deficient embryos does not lead to uniform expression of tll throughout the embryo, as is seen in tor ${ }^{D}$ embryos (Steingrímsson et al. 1991), indicating there must be factors present in the early embryo, in addition to NTF-1, that also repress tll expression. Second, the multimerized 11-bp tor-RE only weakly (if at all) represses expression of the heterologous promoter $d p p$ (data not shown), suggesting that DNA sequences additional to the tor- $\mathrm{RE}$ /and hence the proteins that bind to them) are required for a high level of repression. Third, in our analysis of the minimal tll regulatory region, deletion of the region covered by footprint $\mathrm{C}$, which is adjacent to the tor-RE, results in a slight expansion of the spatial expression pattern, suggesting that a protein binding to this element might be involved in repression. Fourth, the observation that loss-of-function groucho germ-line clones result in embryos with terminal defects (Paroush et al. 1994) suggests that maternally encoded Groucho protein might play a role in $t$ ll regulation. Although Groucho does not bind to DNA (Hartley et al. 1988), it might bind to other proteins regulating tll. Thus, there may be a complex of proteins regulating $t l l$ that responds to the activation of the tor RTK. Consistent with this notion, multiprotein complexes have been implicated in transcriptional activation and repression in both Drosophila and yeast; in addition, the GAGA protein belongs to the Trithorax group of proteins that is thought to be involved in a possible activator complex in Drosophila (Orlando and Paro 1995).

The sevenless (sev) RTK pathway in the Drosophila eye imaginal disc shares many components of the tor RTK pathway, in particular Drk, Sos, Rasl, Raf, and ERKA proteins (for review, see Dickson and Hafen 1994), raising the question of whether any transcription factor targets of these pathways might also be shared. Two ets domain transcription factors, Pointed-P2 and Yan, are targets of the sev pathway and are phosphorylated by ERKA; this phosphorylation converts Pointed-P2 to a transcriptional activator, and inactivates the transcriptional repression function of Yan (Brunner et al. 1994a O'Neill et al. 1994). Also, the Drosophila Jun homolog DJun has been shown to be a target of the sev pathway (Bohmann et al. 1994). Neither ets- nor AP1-binding sites, however, are found in either of the tll minimal regulatory modules, nor does bacterially produced Yan footprint within the proximal regulatory module (G.-J. Liaw, unpubl.).

\section{Multiple tor-REs and graded terminal system activity}

The multiple tor-REs in the proximal and distal tll promoter might play a role in setting the sharp borders of $t l l$ expression. Graded terminal system activity presumably leads to the graded modification and consequent inactivation of the repressor proteins that bind to the tor- $\mathrm{RE}$ (Casanova and Struhl 1989; Pignoni et al. 1992; Sprenger and Nüsslein-Volhard 1992). Graded repressor inactivation in the terminal patterning system would then be analogous to the Bicoid morphogen gradient in the anterior patterning system and the morphogen gradient of nuclear localized Dorsal in the dorsal/ventral patterning system. Just as multiple binding sites for Bicoid and Dorsal in the hunchback and the twist regulatory regions, respectively, appear to be necessary to set sharp, correctly placed borders of expression of these zygotic genes (for review, see Courey and Huang 1995), multiple torREs might similarly help to create distinct, properly positioned borders of $t$ ll transcription.

\section{Materials and methods \\ Construction of tll promoter-reporter fusion genes and germ-line transformation}

Various subfragments of the proximal and distal $t l l$ promoter regions /shown in Fig. 1A, with the exception of construct G21, K.M. Rudolph, G.-J. Liaw, A.J. Courey, and J.A. Lengyel, in prep.) were generated by means of the polymerase chain reaction or by synthesis of the DNA oligonucleotides using Gene Assembler Plus (Pharmacia). The DNA fragments for the G series of constructs were inserted into the PstI site of the vector pAPA2, which is a derivative of pBluescript SK $|+|$ |Stratagene). This vector contains two AvaI sites just flanking the PstI site. These two Aval sites, 5' -CTCGGG-3', were used to multimerize DNA fragments in tandem repeats (Liaw et al. 1993; Liaw 1994). For constructs K12 and K13, multiple copies of the distal region (see Fig. $3 \mathrm{~A}$ ) were inserted into the AvaI site of the vector pAEA5, which is also a derivative of pBluescript $\mathrm{SK} \mid+$ ) (Liaw 1994). For each construct, plasmids were selected that carried four copies of the DNA fragment in question. Constructs G21G27 and G29 (Fig. 4A) were generated by oligonucleotide-directed mutagenesis (Ausubel et al. 1994) using a tetramer of the construct G11 as template. Construct G28 was generated by inserting the fourfold oligomerized region $M-R$ [excised from construct G2 (Liaw et al. 1993) with KpnI and NotI] into the NotI site of pKr672 (Hoch et al. 1990) using NotI linkers. Construct G30 was made by oligonucleotide-directed mutagenesis of a monomer of the construct Gll as template and then multimerization to four copies (Liaw 1994). Construct K13 was generated by oligonucleotide-directed mutagenesis using a tetramer of the construct $\mathrm{K} 12$ as template (see the legend to Fig. 5). DNA sequences of all constructs were then confirmed by DNA sequencing (Sanger et al. 1977). The fourfold multimers were inserted into the multicloning site of the P-element vector PwHZ128, a derivative of PwHZ16 (Liaw et al. 1993). These p-element constructs were injected into $w^{1118}$ embryos as described (Rubin and Spradling 1982; Spradling and Rubin 1982) For each construct, at least six independent lines were established.

Characterization of promoter-driven lacZ expression and endogenous tll expression

Expression of $\beta$-galactosidase in whole embryos was assayed by staining with X-gal (Raghavan et al. 1986). Expression of mRNA of the $l a c Z$ gene and of the endogenous $t l l$ gene was monitored by in situ hybridization (Tautz and Pfeifle 1989) using randomprimed digoxigenin-labeled (Boehringer Mannheim Biochemicals) lac $Z$ DNA and a tll cDNA (Pignoni et al. 1990). Embryonic stages were determined according to Campos-Ortega and Hartenstein (1985). 


\section{Purification of tor-REBS}

Nuclear extracts from 0 - to 12 -hr embryos $\sim \sim 750$ grams $)$ were prepared as described (Soeller et al. 1986) and fractionated on a $50-\mathrm{ml}$ heparin-Sepharose column. Fractions containing tor- $\mathrm{REB}_{\mathrm{a}}$ footprinting activity were eluted with $0.5 \mathrm{M} \mathrm{KCl}$ in HEMG-H [25 mM HEPES (pH 7.6), 0.1 mM EDTA, $12.5 \mathrm{~mm}$ $\mathrm{MgCl}_{2}, 10 \%$ glycerol, and $1 \mathrm{~mm}$ dithiothreitol]. These fractions were pooled, diluted to $0.1 \mathrm{M} \mathrm{KCl}$ in HEMG (like HEMG.H except the concentration of $\mathrm{MgCl}_{2}$ is $2.5 \mathrm{~mm}$ ) and applied to a 20-ml CM-Sepharose column. The column was eluted with 100 $\mathrm{ml}$ of a linear $0.1-0.5 \mathrm{M} \mathrm{KCl}$ gradient in HEMG. Fractions containing tor-REB footprinting activity (eluting between 0.18 and $0.24 \mathrm{M} \mathrm{KCl}$ ) were pooled, supplemented with Nonidet-P40 $\{0.1 \%)$, poly[d(I-C)] $\left\{0.1 A_{260} / \mathrm{ml}\right\}$ and poly[d(A-T) $\left\{0.025 A_{260} /\right.$ $\mathrm{ml}$ ), incubated on ice for $10 \mathrm{~min}$, and centrifuged at $12,000 \mathrm{~g}$ for $10 \mathrm{~min}$. The supernatant, divided into four parts, was applied to four 1-ml DNA affinity columns as described by Kadonaga and Tjian (1986). Two complementary oligonucleotides based on the DNA sequence of footprint $F\left(5^{\prime}\right.$-GATCCGAAGAGTTTACTCAATGCTCAATGAATTCGGAGCGGCGGCGGCAA$3{ }^{\prime}$; Fig. 4D| were used to prepare the DNA affinity resin. The columns were equilibrated with $0.2 \mathrm{M} \mathrm{KCl} \mathrm{HEMGN} \mathrm{(HEMG}$ containing $0.1 \%$ Nonidet-P 40). The columns were washed with $0.25 \mathrm{M} \mathrm{KCl} \mathrm{HEMGN}$ and the tor $-\mathrm{REB}_{\mathrm{a}}$ was eluted at $0.4 \mathrm{M} \mathrm{KCl}$.

The procedure for the purification of the second tor-REB (tor$R E B b$ ) was identical to that described by Huang et al. (this issue) for purification of the DREB, with the exception that the DNA affinity column used was the tor-RE the DNA sequence of footprint D, 5'-GATCGCTGCGTTCCTTGCTCAATGAATTTTTCGCAATGGCAGCCTG-3') instead of the DRE (Huang et al. 1995)

For DNase I protection experiments (Kadonaga et al. 1987), DNA fragments were end-labeled with $\left(\gamma^{-}{ }^{32} \mathrm{P}\right)$ ATP and T4 polynucleotide kinase (Sambrook et ai. 1989 ).

\section{Purification of GAGA and GST-NTF-1}

GAGA protein was purified from 0 - to 12 -hr embryonic nuclear extract by a simplification of the procedure of Biggin et al. (1988). Nuclear extract was loaded onto a $90 \mathrm{ml}$ of SP-Sepharose column. Fractions containing GAGA footprinting activity, eluting at $0.35 \mathrm{M} \mathrm{KCl} \mathrm{HEMG}$, were pooled, supplemented with NP. 40 to $0.1 \%, 0.15 A_{260} / \mathrm{ml}$ of poly[d(I-C) $]$ and $0.05 A_{260} / \mathrm{ml}$ of poly[d(A-T)], and purified by DNA affinity chromatography as described by Biggin et al. (1988).

GAGA was also purified from GAGA-expressing Escherichia coli BL21(DE3)pLysE containing the plasmid pARGAGA a generous gift of W. Soeller, Pfizer, Inc., Groton, CT); purification was as described by Lu et al. (1993), using LB medium and HEMG buffer instead of NZCYM medium and HEG buffer.

GST-NTF-1 was purified from expressing E. coli DH $5 \alpha$. NTF-1 cDNA (from R. Tiian) (Dynlacht et al. 1989) was cloned into the expression vactor pGEX-2T (Pharmacia). The GSTNTF-1 fusion protein was purified using glutathione-agarose (Sigma) chromatography (Smith and Johnson 1988). To remove degradation products, the affinity-purified protein was concentrated to $0.5 \mathrm{ml}$, dialyzed against $0.1 \mathrm{M} \mathrm{KCl} \mathrm{HEMGN} \mathrm{overnight}$ and then chromatographed on a 50-ml Sephacryl S-300 (Pharmacia) column in $0.1 \mathrm{M} \mathrm{KCl} \mathrm{HEMGN}$. The first $2 \mathrm{ml}$ of included volume from this column was $75 \%$ intact GST-NTF-1.

Protein concentrations were determined by the Bradford assay (Bio-Rad, Inc).

Western blotting using anti-GAGA antibodies

Proteins were loaded onto duplicate $9 \%$ polyacrylamide-SDS gels. Proteins in one gel were detected by silver staining (Ausubel et al. 1994l, whereas proteins in the other gel were blotted to a nitrocellulose filter using a Nova Blot apparatus (model LKB 2117/250; Pharmacia). Incubation with antibody and detection of proteins on the nitrocellulose filter followed the ImmunoBlot Kit (Bio-Rad, Inc) protocol. The rat polyclonal anti-GAGA antibody $(\alpha 519)$ was generously provided by C. Benyajati (University of Rochester, NY|. Molecular mass markers were from Sigma Chemical Co. and GIBCO; the sizes shown are the apparent molecular masses given by the supplier.

\section{Phosphorylation of the NTF-1 protein by MAPK}

Rat ERK2 $\left(p 42^{\text {mank }}\right.$ and constitutively active rabbit MAPKK were generous gifts of T. Haystead (University of Virginia, Charlottesville); phosphorylation was carried out as described by Haystead et al. (1992). ERK2 was activated by MAPKK in activation buffer $150 \mathrm{~mm} \quad \beta$-glycerophosphate $(\mathrm{pH} 7.2), 1.5 \mathrm{~mm}$ EGTA, $0.15 \mathrm{mM}$ sodium orthovanadate, $1 \mathrm{mM}$ DTT, $7.5 \mathrm{~mm}$ $\mathrm{MgCl}_{2}$, and $300 \mu \mathrm{M} \mathrm{ATP} \mid$ for $90 \mathrm{~min}$ at $30^{\circ} \mathrm{C}$. Activated MAPK was dialyzed against activation buffer without $\mathrm{Mg}^{+2}$ and ATP. Aliquots $(10 \mu \mathrm{l})$ of activated MAPK were then incubated with either various proteins in activation buffer (with $100 \mu \mathrm{M}$ $\mid \gamma{ }^{32}$ P|ATP rather than $300 \mu \mathrm{M}$ ATP $\mid$. Myelin basic protein was obtained from Sigma Chemical Co. After electrophoresis of the reaction products on a $10 \%$ polyacrylamide-SDS gel, phosphorylated proteins were detected by autoradiography. Incorporation of ${ }^{32} \mathrm{P}$ into various bands was quantitated with a PhosphorImager (Molecular Dynamics Inc.)

\section{Fly stocks and germ-line clones}

Two grainyhead $(g r h)$ mutant lines, $d p g r h^{B 32} \mathrm{cn} b w / C y O$ and $d p g r h^{B .37} \mathrm{cn}$ bw $\mathrm{CyO}$, were obtained from $\mathrm{S}$. Bray (University of Cambridge, UK!. The dominant female sterile mutation Fs/2)D, which like grh maps to the right arm of the second chromosome (Schüpbach 1982), was obtained from T. Schüpbach; this chromosome was balanced with $S^{1} S p^{1}$ Ms(2)M1 $b w^{\prime \prime} C y O$ lobtained from the Bloomington stock center). Germ-line clones were generated by X-irradiation of first instar larvae from $d p$ grh $c n b w$ : $C y O$ females crossed with $F_{S}(2) D /$ CyO males (Perrimon and Gans 1983); adult female progeny potentially carrying germ-line clones (i.e., $d p \mathrm{grh} \mathrm{cn} \mathrm{bw/Fs(2)D)}$ were mated in groups of 10 with $g r h / C y O$ males. Groups of females producing eggs with normal chorionic appendages [indicating the presence of homozygous grh germ-line clones, as germ-line cells carrying Fs(2)D produce eggs with abnormal or no dorsal appendages [Schüpbach 1982)] were used for egg collections.

The alleles $T_{r} 1^{1.36}, \operatorname{Tr} l^{102}$, and $T r l^{R 85}$ were obtained from $\mathrm{F}$. Karch (Universite de Geneve, Switzerland). $T_{r} l^{62}$ and $T_{r} l^{R 85}$ were placed over the ovo ${ }^{11}$ FRT chromosome lobtained from the Bloomington stock center) and recombination induced with the heat shock FLP according to the protocol of Chou et al. (1993) but no clones were obtained. We also sought to generate germ-line clones by X-irradiation of $\mathrm{Tr}^{13 C^{1} / O \mathrm{VO}^{D 1}}$ as described above, but again, no such clones were obtained.

\section{Acknowledgments}

We thank I. R. Lehman for providing Drosophila embryos in our initial purification of tor-REBs, S. Bray, F. Karch, T. Schüpbach, and the Bloomington Stock Center for providing mutants, C. Benyajati, T. Haystead, and R. Tjian for proteins and antibodies, C.-S. Chiang for suggestions in the protein purification, and J.O. $\mathrm{Vu}$ for help with germ-line transformation and in situ hybridization. This work was supported by the American Cancer So- 
ciety grant NP-785 and National Institutes of Health (NIH grant GM51270 to J.A.L and A.J.C., NIH grant GM44522 to A.J.C., and NIH grant HD09948 to J.A.L.

The publication costs of this article were defrayed in part by payment of page charges. This article must therefore be hereby marked "advertisement" in accordance with 18 USC section 1734 solely to indicate this fact.

\section{References}

Attardi, L.D. and R.Tiian. 1993. Drosophila tissue-specific tran scription factor NTF-1 contains a novel isoleucine-rich activation motif. Genes \& Dev. 7: 1341-1353.

Ausubel, F.M., R. Brent, R.E. Kingston, D.D. Moore, J.G. Seidman, J.A. Smith, and K. Struhl. 1994. Current protocols in molecular biology, vol. 1. John Wiley \& Sons, New York.

Biggin, M., S. Bickel, M. Benson, V. Pirrotta, and R. Tiian. 1988. Zeste encodes a sequence-specific transcription factor that activates the Ultrabithorax promoter in vitro. Cell 53: $713-$ 722.

Bohmann, D., M.C. Ellis, L.M. Staszewski, and M. Mlodzik. 1994. Drosophila Jun mediates Ras-dependent photoreceptor determination. Cell 78: 973-986.

Bray, S.J. and F.C. Kafatos. 1991. Developmental function of Elf-1: An essential transcription factor during embryogenesis in Drosophila. Genes \& Dev. 5: 1672-1683.

Bray, S.J., B. Burke, N.H. Brown, and J. Hirsh. 1989. Embryonic expression pattern of a family of Drosophila proteins that interact with a central nervous system regulatory element. Genes \& Dev. 3: 1130-1145.

Brönner, G. and H. Jäckle. 1991. Control and function of terminal gap gene activity in the posterior pole region of the Drosophila embryo. Mech. Dev. 35: 205-211.

Brönner, G., Q. Chu-LaGraff, C.Q. Doe, B. Cohen, D. Weigel, H. Taubert, and H. Jäckle. 1994. Spl/egr-like zinc-finger protein required for endoderm specification and germ-layer formation in Drosophila. Nature 369: 664-668

Brunner, D., K. Ducker, N. Oellers, E. Hafen, H. Scholz, and C. Klämbt. 1994a. The ETS domain protein pointed-P2 is a target of MAP kinase in the sevenless signal transduction pathway. Nature 370: 386-389.

Brunner, D., N. Oellers, J. Szabad, W.H. Biggs III, S.L. Zipursky, and E. Hafen. 1994b. A gain of function mutation in Drosophila MAP kinase constitutively activates multiple receptor tyrosine kinase signalling pathways. Cell 76: 875-888.

Campos-Ortega, J.A. and V. Hartenstein. 1985. The embryonic development of Drosophila melanogaster. Springer-Verlag, Berlin, Heidelberg, Germany.

Casanova, J. and G. Struhl. 1989. Localized surface activity of torso, a receptor tyrosine kinase, specifies terminal body pattern in Drosophila. Genes \& Dev. 3: 2025-2038.

Chou, T.B., E. Noll, and N. Perrimon. 1993. Autosomal P $\left[o^{D}{ }^{D}\right]$ dominant female-sterile insertions in Drosophila and their use in generating germ-line chimeras. Development 119: 1359-1369.

Courey, A.J. and J.D. Huang. 1995. The establishment and interpretation of transcription factor gradients in the Drosophila embryo. Biochim. Biophys. Acta 1261: 1-18.

Dickson, B. and E. Hafen. 1994. Genetics of signal transduction in invertebrates. Curr. Opin. Genet. Dev. 4: 64-70.

Dynlacht, B.D., L.D. Attardi, A. Admon, M. Freeman, and R. Tijian. 1989. Functional analysis of NTF-1, a developmentally regulated Drosophila transcription factor that binds neuronal cis elements. Genes \& Dev. 3: 1677-1688.

Erickson, A.K., D.M. Payne, P.A. Martino, A.J. Rossomando, J.
Shabanowitz, M.J. Weber, D.F. Hunt, and T.W. Sturgill. 1990. Identification by mass spectrometry of threonine 97 in bovine myelin basic protein as a specific phosphorylation site for mitogen-activated protein kinase. 1. Biol. Chem. 265: 19728-19735

Farkas, G., J. Gausz, M. Galloni, G. Reuter, H. Gyurkovics, and F. Karch. 1994. The Trithorax-like gene encodes the Drosophila GAGA factor. Nature 371: 806-808.

Hartley, D.A., A. Preiss, and S. Artavanis-Tsakonas. 1988. A deduced gene product from the Drosophila neurogenic locus, Enhancer of split, shows homology to mammalian G-protein $\beta$ subunit. Cell 55: 785-795.

Haystead, T.A., P. Dent, J. Wu, C.M. Haystead, and T.W. Sturgill. 1992. Ordered phosphorylation of $\mathrm{p}^{4} 2^{\mathrm{mapk}}$ by MAP kinase kinase. FEBS Lett. 306: 17-22.

Hill, C.S. and R. Treisman. 1995. Transcriptional regulation by extracellular signals: Mechanisms and specificity. Cell 80: 199-211.

Hoch, M., C. Schroder, E. Seifert, and H. Jäckle. 1990. cis-acting control elements for Krüppel expression in the Drosophila embryo. EMBO 1. 9: 2587-2595.

Hoch, M., N. Gerwin, H. Taubert, and H. Jäckle. 1992. Competition for overlapping sites in the regulatory region of the Drosophila gene Krüppel. Science 256: 94-97.

Huang, J.-D., T. Dubnicoff, G.-J. Liaw, Y. Bai, S. Valentine, J.M. Shirokawa, J.A. Lengyel, and A.J. Courey. 1995. Binding sites for transcription factor NTF-1 contribute to the ventral repression of decapentaplegic. Genes \& Dev. this issue.

Kadonaga, I.T. and R. Tiian. 1986. Affinity purification of sequence-specific DNA binding proteins. Proc. Natl. Acad. Sci. 83: 5889-5893.

Kadonaga, J.T., K.R. Carner, F.R. Masiarz, and R. Tjian. 1987. Isolation of cDNA encoding transcription factor $\mathrm{Spl}$ and functional analysis of the DNA binding domian. Cell 51: 1079-1090.

Kerrigan, L.A., G.E. Croston, L.M. Lira, and J.T. Kadonaga. 1991. Sequence-specific transcriptional antirepression of the Drosophila Krüppel gene by the GAGA factor. I. Biol. Chem. 266: 574-582.

Liaw, G.-J. 1994. Improved protocol for directional multimerization of DNA fragment. Biotechniques 17: 668-670.

Liaw, G.-J. and J.A. Lengyel. 1992. Control of tailless expression by bicoid, dorsal and synergistically interacting terminal system regulatory elements. Mech. Dev. 40: 47-61.

Liaw, G.-J., E. Steingrímsson, F. Pignoni, A.J. Courey, and J.A. Lengyel. 1993. Characterization of downstream elements in a Raf-1 pathway. Proc. Natl. Acad. Sci. 90: 858-862.

Lu, Q., L.L. Wallrath, H. Granok, and S.C. Elgin. 1993. (CT) $(G A)_{n}$ repeats and heat shock elements have distinct roles in chromatin structure and transcriptional activation of the Drosophila hsp26 gene. Mol. Cell. Biol. 13: 2802-2814.

O'Brien, T., R.C. Wilkins, C. Giardina, and J.T. Lis. 1995. Distribution of GAGA protein on Drosophila genes in vivo. Genes \& Dev. 9: 1098-1110.

O'Neill, E.M., I. Rebay, R. Tjian, and G.M. Rubin. 1994. The activities of two Ets-related transcription factors required for Drosophila eye development are modulated by the Ras/ MAPK pathway. Cell 78: 137-147.

Orlando, V. and R. Paro. 1995. Chromatin multiprotein com plexes involved in the maintenance of transcription pat terns. Curr. Opin. Genet. Dev. 5: 174-179.

Paroush, Z., R. Finley Jr., T. Kidd, S.M. Wainwright, P.W. Ingham, R. Brent, and D. Ish-Horowicz. 1994. Groucho is required for Drosophila neurogenesis, segmentation, and sex determination and interacts directly with hairy-related bHLH proteins. Cell 79: 805-815. 
Perrimon, N. 1993. The torso receptor protein-tyrosine kinase signaling pathway: An endless story. Cell 74: 219-222.

Perrimon, N. and M. Gans. 1983. Clonal analysis of the tissue specificity of recessive female-sterile mutations of Drosophila melanogaster using a dominant female-sterile mutation Fs $|1| \mathrm{K} 1237$. Dev. Biol. 100: 365-373.

Pignoni, F., R.M. Baldarelli, E. Steingrimsson, R.J. Diaz, A. Patapoutian, J.R. Merriam, and I.A. Lengyel. 1990. The Drosophila gene tailless is expressed at the embryonic termini and is a member of the steroid receptor superfamily. Cell 62: 151-163.

Pignoni, F., E. Steingrímsson, and J.A. Lengyel. 1992. bicoid and the terminal system activate tailless expression in the early Drosophila embryo. Development 115: 239-251.

Raghavan, K.V., M.A. Crosby, P.H. Mathers, and E.M. Meyerowitz. 1986. Sequences sufficient for correct regulation of Sgs-3 lie close to or within the gene. EMBO J. 5: 3321-3326.

Roberts, S.G.E. and M.R. Green 1995. Dichotomous regulators. Nature 375: 105-106.

Rubin, G.M. and A.C. Spradling. 1982. Genetic transformation of Drosophila with transposable element vectors. Science 218: 348-353.

Sambrook, J., E.F. Fritsch, and T. Maniatis. 1989. Molecular cloning: A laboratory manual. Cold Spring Harbor Laboratory Press, Cold Spring Harbor, New York.

Sanger, F., S. Nicklen, and A.R. Coulson. 1977. DNA sequencing with chain-terminating inhibitors. Proc. Natl. Acad. Sci. 74: 5463-5467.

Schüpbach, T. 1982. Autosomal mutations that interfere with sex determination in somatic cells of Drosophila have no direct effect on the germline. Dev. Biol. 89: 117-127.

Smith, D.B. and K.S. Johnson. 1988. Single-step purification of polypeptides expressed in Escherichia coli as fusions with glutathione S-transferase. Gene 67: 31-40.

Soeller, W., S.J. Poole, and T. Kornberg. 1986. In vitro transcription of the Drosophila engrailed gene. Genes \& Dev. 2: 6881.

Soeller, W.C., C.E. Oh, and T.B. Kornberg. 1993. Isolation of cDNAs encoding the Drosophila GAGA transcription factor. Mol. Cell. Biol. 13: 7961-7970.

Spradling, A.C. and G.M. Rubin. 1982. Transposition of cloned p-elements into Drosophila germ line chromosomes. Science 218: 341-347.

Sprenger, F. and C. Nüsslein-Volhard. 1992. torso receptor activity is regulated by a diffusible ligand produced at the extracellular terminal regions of the Drosophila egg. Cell 71: 987-1001.

Steingrímsson, E. 1992. "Structure and function of the zygotic terminal gene tailless." Ph.D. thesis, University of California, Los Angeles, Los Angeles, CA.

Steingrímsson, E., F. Pignoni, G.J. Liaw, and J.A. Lengyel. 1991. Dual role of the Drosophila pattern gene tailless in embryonic termini. Science 254: 418-421.

Strecker, T., K. Kongsuwan, J.A. Lengyel, and J.R. Merriam. 1986. The zygotic mutant tailless affects the anterior and posterior of the Drosophila embryo. Dev. Biol. 113: 64-76.

Tautz, D. and C. Pfeifle. 1989. A non-radioactive in situ hybridization method for the localization of specific RNAs in Drosophila embryos reveals translational control of the segmentation gene hunchback. Chromosoma 98: 81-85.

Throckmorton, L.H. 1975. The phylogeny, ecology, and geography of Drosophila. In Handbook of genetics (ed. R.C. King), pp. 421-469. Plenum, New York.

Tsukiyama, T., P.B. Becker, and C. Wu. 1994. ATP-dependent nucleosome disruption at a heat-shock promoter mediated by binding of GAGA transcription factor. Nature 367: 525-
532

Weigel, D., G. Jurgens, M. Klingler, and H. Jäckle. 1990. Two gap genes mediate maternal terminal pattern information in Drosophila. Science 248: 495-498. 


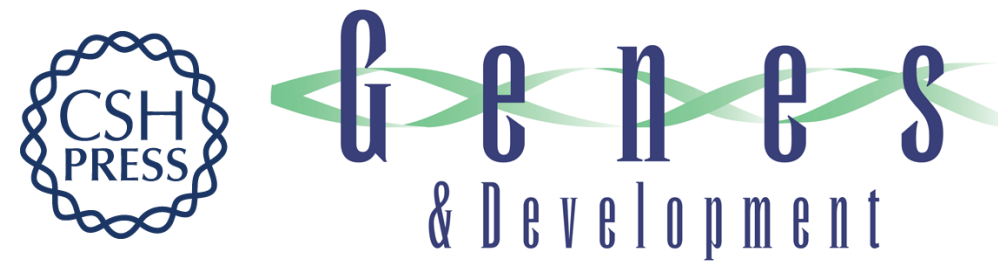

\section{The torso response element binds GAGA and NTF-1/Elf-1, and regulates tailless by relief of repression.}

G J Liaw, K M Rudolph, J D Huang, et al.

Genes Dev. 1995, 9:

Access the most recent version at doi:10.1101/gad.9.24.3163

References This article cites 51 articles, 20 of which can be accessed free at:

http://genesdev.cshlp.org/content/9/24/3163.full.html\#ref-list-1

License

Email Alerting

Service

Receive free email alerts when new articles cite this article - sign up in the box at the top right corner of the article or click here.

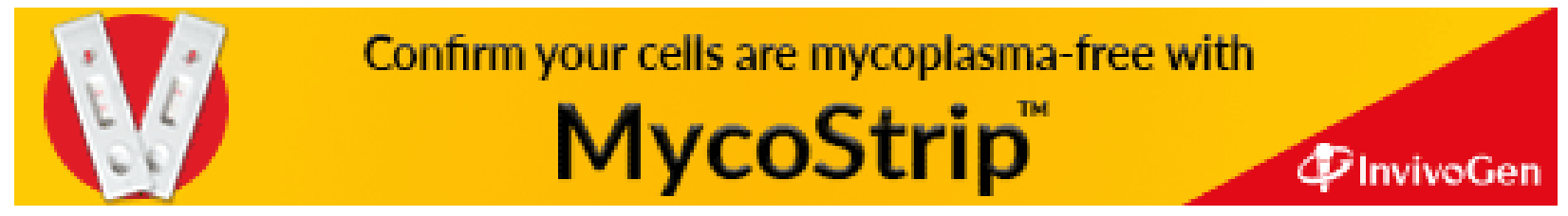

